\title{
Magnetic origin of black hole winds across the mass scale
}

\author{
Keigo Fukumura ${ }^{1 \star}$, Demosthenes Kazanas ${ }^{2}$, Chris Shrader ${ }^{2,3}$, Ehud Behar ${ }^{4,5}$, Francesco Tombesi ${ }^{2,5,6}$ and \\ loannis Contopoulos ${ }^{7}$
}

\begin{abstract}
Invariably, black hole accretion disks seem to produce plasma outflows that result in blue-shifted absorption features in their spectra'. The X-ray absorption-line properties of these outflows are diverse, ranging in velocity from non-relativistic ${ }^{2}$ $\left(\sim 300 \mathrm{~km} \mathrm{~s}^{-1}\right)$ to sub-relativistic ${ }^{3}(\sim 0.1 \mathrm{c}$, where $c$ is the speed of light) and a similarly broad range in the ionization states of the wind plasma ${ }^{2,4}$. We report here that semi-analytical, self-similar magnetohydrodynamic wind models that have successfully accounted for the $X$-ray absorber properties of supermassive black holes ${ }^{5,6}$ also offer a good fit to the highresolution X-ray spectrum of the accreting stellar-mass black hole GRO J1655-40. This provides an explicit theoretical argument of their magnetohydrodynamic origin (aligned with earlier observational (claims) ${ }^{7}$ and supports the notion of a universal magnetic structure of the observed winds across all known black hole sizes.
\end{abstract}

The importance of the plasma outflows (winds) from black hole accretion disks lies partly in the fact that they may remove the accretion disks' angular momentum ${ }^{8}$, a condition necessary for making accretion possible, and partly in their potential feedback and influence on their environment ${ }^{9}$. The variety of processes that can launch such winds ${ }^{10-16}$ has made their origin contentious. However, X-ray spectroscopic data and analysis ${ }^{7,17-19}$ of the wind associated with the X-ray binary (XRB) GRO J1655-40 have argued in favour of a magnetic origin, but only by excluding the other candidate processes. Here, we present first-principles computations of absorption-line spectra of self-similar magnetohydrodynamic (MHD) models for the accretion disk wind ${ }^{5,6}$ that reproduce both the combined global ionization properties and the detailed kinematic structure of the absorption features of GRO J1655-40. Most importantly, these wind models are the same as those that have accounted successfully for the X-ray absorber systematics of active galactic nuclei (AGNs) as diverse as Seyfert galaxies ${ }^{5,20}$ and broad-absorption-line (BAL) quasars ${ }^{6}$, modified only by the different ionizing spectrum of GRO J1655-40 and scaled to its black hole mass. This demonstrates a universality of properties for accretion disk winds across the entire mass range $\left(10 M_{\odot}-10^{9} M_{\odot}\right.$, where $M_{\odot}$ is solar mass) for black holes ${ }^{21}$.

The earlier observational claims favouring the magnetic origin of the GRO J1655-40 winds were made by excluding the other two plausible mechanisms for launching winds off accretion disks on the basis of the observed line properties of this system ${ }^{7,17}$. The first of these is thermally driven winds ${ }^{10,16}$, launched by heating the disk surface by the X-rays produced near the compact object to the Compton temperature $T_{\mathrm{C}}$. For a sufficiently large distance $R_{\mathrm{C}}$ along the disk, the plasma thermal velocity $V_{\text {th }}$ can be larger than the local escape velocity $V_{\mathrm{e}}, k T_{\mathrm{C}} / m_{\mathrm{p}} \approx V_{\text {th }}^{2}>V_{\mathrm{e}}^{2} \approx G M / R_{\mathrm{C}}$ (where $G$ is the gravitational constant and $M, m_{\mathrm{p}}$ are the black hole and proton masses respectively), allowing escape of the X-ray heated matter to infinity. The GRO J1655-40 quasi-thermal disk spectrum of $k T \approx 1.34 \mathrm{keV}$, then, implies ${ }^{2} R_{\mathrm{C}}>7 \times 10^{12} \mathrm{~cm}$, larger than the binary orbit and grossly inconsistent with the photoionized wind properties (however, see other views ${ }^{22-24}$ ). The second possible mechanism is winds driven by radiation pressure (at sub-Eddington luminosity, $L<L_{\mathrm{Edd}}$ ); these rely on the increased line pressure on partially ionized elements, in analogy with stellar winds ${ }^{25}$. However, as also indicated earlier ${ }^{7}$, the high values of the ionization parameter $\xi=L_{\mathrm{X}} /\left(n r^{2}\right)$ (where $L_{\mathrm{X}}$ is the ionizing luminosity, $n$ the plasma density and $r$ the radial distance from the ionizing source) implied by the X-ray continuum and line luminosity of GRO J1655-40 $\left(\xi>10^{3}\right)$ argue for an over-ionized wind plasma, thereby excluding line-pressure-driven winds ${ }^{26}$.

Winds can also be launched with the help of magnetic fields, either in combination with the disk centrifugal forces ${ }^{12,13,15}$ or by the vertical magnetic pressure gradients in the disk ${ }^{14}$. Their morphological distinction from those launched by the other two processes lies in their inherently 2D structure, as they are launched across the entire disk domain. Because of the large dynamic range of their launching radii $\left(R_{\text {disk }} / R_{\mathrm{S}} \approx 10^{6}\right.$, where $R_{\mathrm{S}}$ is the Schwarzschild radius), they are best described by self-similar models with their density and velocity spanning similarly large dynamic ranges. This fact is consistent with the wide $\xi$-range of the ions observed in the X-ray absorption line spectra of AGN. Self-similarity allows separation of the wind density $n(r, \theta)$ in $r$ (the spherical radial coordinate) and $\theta$ (the disk polar angle): that is, $n(r, \theta)=n_{0} f(\theta)\left(r / r_{0}\right)^{-(1+\alpha)}$ with $^{5} n_{0} \propto \dot{m} / M$ ( $M$ is the black hole mass and $\dot{m} \equiv \dot{M} / \dot{M}_{\mathrm{Edd}}, M_{\mathrm{Edd}}=L_{\mathrm{Edd}} / c^{2}$ ) and $f(\theta) \approx \mathrm{e}^{5(\theta-\pi / 2)}$, giving these winds a poloidal appearance (see Fig. 1). Note here that $n_{0}$ is the wind density at the innermost launching radius at $r=r_{0}$. With $r$ measured in units of the Schwarzschild radius $R_{\mathrm{S}}(\propto M)$, the wind column $N_{\mathrm{H}} \propto n(r) r \propto r^{-\alpha}$ is independent of the mass $M$, as also are $\xi \propto L / n(r) r^{2} \propto r^{-(1-\alpha)}$ (assuming $L \propto \dot{m} M$ ) and $V / c \propto r^{-1 / 2}$, with $n_{0}$ and $\alpha$ the main free model parameters. The computed radial profiles of $N_{\mathrm{H}}, V$ and $\xi$ for $\alpha=0.2$ are shown in Fig. 2.

With the physical parameters that determine the winds' spectroscopic properties, $N_{\mathrm{H}}, V$ and $\xi$, independent of the compact object mass, $M$, these models are equally well applicable to AGNs and XRBs, the greatest difference between these two classes being the spectral energy distribution (SED) of ionizing radiation ${ }^{6}$. Eliminating $r$ between the hydrogen equivalent column of an ion,

James Madison University, 800 South Main Street, Harrisonburg, Virginia 22807, USA. ${ }^{2}$ NASA, Goddard Space Flight Center, 8800 Greenbelt Road, Greenbelt, Maryland 20771, USA. ${ }^{3}$ Universities Space Research Association, 10211 Wincopin Circle, Suite 500, Columbia, Maryland 21044, USA. ${ }^{4}$ Physics Department, Technion - Israel Institute of Technology, Haifa 3200003, Israel. ${ }^{5}$ Astronomy Department, University of Maryland, College Park, Maryland 20742, USA. ${ }^{6}$ Dipartimento di Fisica, Universita'di Roma Tor Vergata, Via della Ricerca Scientifica 1, I-00133 Roma, Italy. ${ }^{7}$ Academy of Athens, Soranou Efesiou 2, GR 11527 Athens, Greece. *e-mail: fukumukx@jmu.edu 


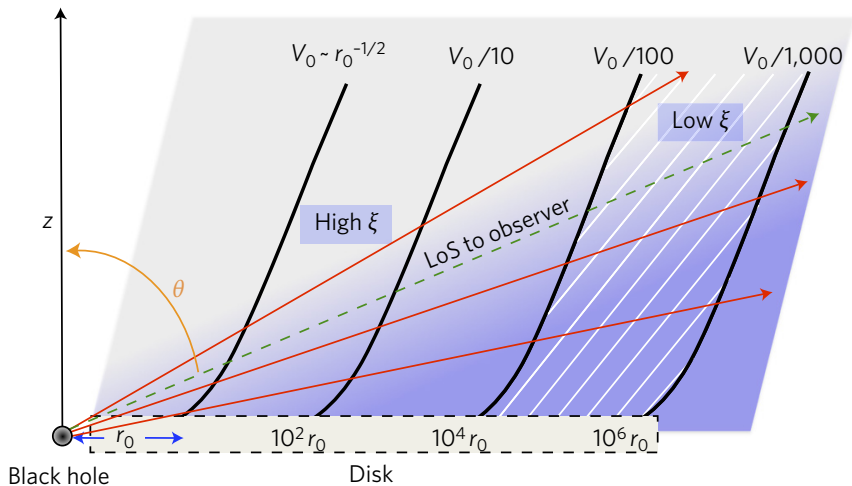

Figure 1 |Schematic of MHD accretion disk wind. Poloidal two-dimensional wind streamlines (thick solid grey lines), the decreasing velocity $\left(V_{0} \approx c\right)$ and ionization $(\xi)$ with radius is illustrated. The hatched region represents the absorbing region of the GRO J1655-40 wind with velocity $100-1,000 \mathrm{~km} \mathrm{~s}^{-1}$. The red arrows indicate possible lines of sight (LoS), with the green arrow believed to be the true line of sight based on published binary solutions.

$N_{\mathrm{H}}$, and its $\xi$-value, we obtain $N_{\mathrm{H}}(\xi) \propto \xi^{\alpha /(1-\alpha)}$, the absorption measure distribution $(\mathrm{AMD})^{4,27}$; this relation can provide the wind density radial profile (that is, the value of $\alpha$ ) by observations of absorption line properties alone. The crucial test of any wind model is then to produce the correct values of $N_{\mathrm{H}}$ and $V$ for the values of $\xi$ at which the observed ions are present, for all ionic species. Most works ${ }^{17,28}$ modelling the X-ray absorbers of GRO J1655-40 focus on accurately fitting the Fe Xxv and Fe xxvi profiles to obtain the local values of $N_{\mathrm{H}}, \xi$ and $V$. In contrast, our approach uses an established MHD wind model, and aims, by reproducing the combined ionization/kinematic properties of the ensemble of observed transitions, to determine its global parameters $n_{0}$ and $\alpha$.

We have computed the photoionization structure of self-similar MHD winds following our own procedure ${ }^{5,6,20}$ with the input continuum spectrum being a multicolour disk of innermost temperature ${ }^{17}$ $k T=1.34 \mathrm{keV}$. Because the procedure is computationally intensive, $\alpha$ and $n_{0}$ were not varied independently but in synchrony so that they produce an AMD consistent broadly with observations (see further discussion in Methods). We have thus used a grid of representative values for $\alpha$ (as listed in Supplementary Table 1) for which the broad-band fitting yields the best-fit density normalization of $\tilde{n}_{17}=9.3$ (or $n_{0} \approx 10^{18} \mathrm{~cm}^{-3}$ ) with $\alpha=0.2$ wind. In Fig. 3 we show the results of these calculations that provide the bestfit spectrum between $1.5 \AA$ and $12.2 \AA$, based on the comparison between modelled and observed equivalent width for major lines analysed in this work (see the discussion in Methods and Supplementary Fig. 4).

Our photoionization calculations split the radial coordinate $r$ along the line of sight into six to seven slabs per decade with $\Delta r / r \simeq 0.15$ and use XSTAR ${ }^{29}$ to compute the local ionic abundances and opacities; these are then used to compute the transfer of radiation through each slab, with the output used as input in the next one. In such treatments, one typically introduces artificial turbulent broadening of the resulting lines by $\sim 500 \mathrm{~km} \mathrm{~s}^{-1}$ through the parameter vturb of XSTAR. Note that no such broadening is necessary in our models; instead, the lines are naturally broadened by the velocity shear of adjacent wind layers. The profiles of all lines shown in Fig. 3 were computed by considering the continuous absorption of radiation by each ion as its ionic fraction and velocity vary with $r$ along the observer's line of sight. As shown in Fig. 4, the profiles begin shallow, broad and highly blue-shifted at the smallest radii where a given ion is formed. As $r$ increases, larger ionic fraction and lower velocity make the lines less blue-shifted and deeper, to achieve

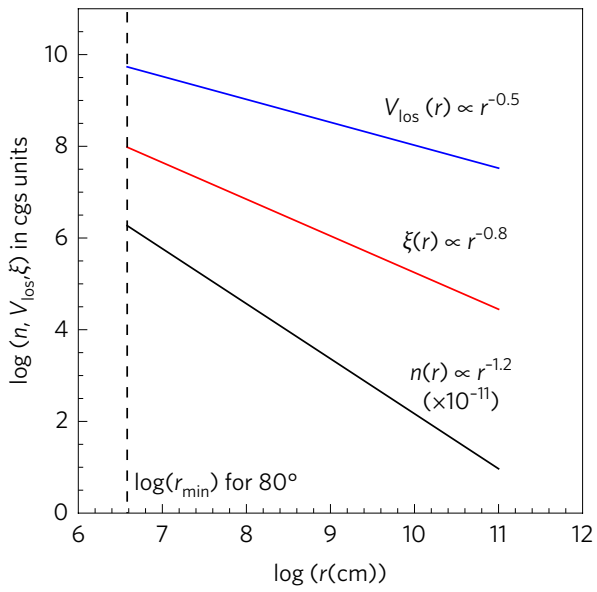

Figure $\mathbf{2}$ | Radial wind profiles inferred from our best-fit model of $\alpha=0.2$. Wind density $n\left(\mathrm{~cm}^{-3}\right)$ (black), the line-of-sight wind velocity $V_{\text {los }}\left(\mathrm{cm} \mathrm{s}^{-1}\right)$ (blue) and the ionization parameter $\xi\left(\mathrm{erg} \mathrm{cm} \mathrm{s}^{-1}\right)$ (red) are shown for $\theta_{\text {obs }}=80^{\circ}$ and $\alpha=0.2$. The vertical dashed line indicates the location of the innermost wind streamline for $80^{\circ}$. Note that $\log (n)$ is offset by -11.0 in the vertical direction for presentation purposes.

their final shape at distances where $\xi$ becomes too small to support that ion. One should finally note that these calculations use only the radial component of the wind velocity, which depends on the observer's inclination angle ${ }^{14}$.

The broader behaviour of our models - that is, the relations among $\left(N_{H}, \xi, V\right)$ where $N_{H} \propto \xi^{\alpha /(1-\alpha)}, N_{H} \propto V^{2 \alpha}$ and $V \propto \xi^{1 / 2(1-\alpha)}-$ is a result of their self-similarity (compare with $N_{\mathrm{H}} \propto \xi^{-3}$ of a specific thermal wind $\left.\operatorname{mode}^{23}\right)$. The Chandra grating observations of GRO J1655-40 (and also those of AGN) indicate a weak dependence of $N_{\mathrm{H}}$ on $\xi(\alpha \approx 0.2 \ll 1)$. This behaviour is consistent with observations: the high $-\xi$ transitions of $\mathrm{Fe}, \mathrm{Ni}$ and $\mathrm{Co}$ are systematically broader and of higher velocity, of order $V \approx 100-1,000 \mathrm{~km} \mathrm{~s}^{-1}$ (implying $r \lesssim 10^{4} R_{S}$ ), with $N_{\mathrm{H}} \approx 10^{23} \mathrm{~cm}^{-2}$. The lower- $\xi$ X-ray absorbers, on the other hand, are narrower and slower $\left(V \approx 100 \mathrm{~km} \mathrm{~s}^{-1}\right)$, implying larger distance from the black hole, with lower $N_{\mathrm{H}}\left(\sim 10^{22} \mathrm{~cm}^{-2}\right)$. Detailed best-fit wind properties are listed in Supplementary Table 2.

As discussed elsewhere ${ }^{21}$, the ionization structure of the wind plasma is independent of the compact object mass $M$, once radii are scaled by $R_{\mathrm{S}}$ and the mass flux by $\dot{M}_{\text {Edd. }}$. For a given (normalized) mass flux $\dot{m}$, differences in the wind properties are reduced to different values of the inclination angle $\theta$ and ionizing SED. Progressively increasing (decreasing) the X-ray contribution of the SED leads to decreasing (increasing) absorber's velocities ${ }^{6}$. As such, the $V \approx 100-1,000 \mathrm{~km} \mathrm{~s}^{-1}$ of the H/He-like Fe absorbers of (the X-ray-dominated) GRO J1655-40 is considerably smaller than those $\left(V \approx 10^{4} \mathrm{~km} \mathrm{~s}^{-1}\right)$ of Seyfert galaxies ${ }^{3}$, whose SEDs are dominated by the big blue bump; in agreement with this notion, BAL quasars, with the weakest X-ray contribution in their SED amongst AGN, exhibit the highest absorber's velocities ${ }^{6}$. Another issue associated with the parameters of these winds is the presence of Fe XxII lines at $11.75,11.9 \AA$, as these are thought to be high density indicators ${ }^{10}$. We have estimated that these lines can also be produced by photon excitation near the edge of our model winds, a fact consistent with their observed velocities; this issue will be treated in more detail in future work. Finally, another qualitative difference in the absorber's properties of GRO J1655-40 from those of AGNs is the absence of low $\xi\left(\lesssim 10^{2.5}\right)$ absorbers in GRO J1655-40, attributed to the spatial extent of its wind, limited by the size of the binary orbit $\left(r \gtrsim 10^{12.5} \mathrm{~cm}, V \lesssim 250 \mathrm{~km} \mathrm{~s}^{-1}\right)$.

Finally, the radial density and velocity of these winds with radius indicate that their (normalized) mass flux $\dot{m} \approx r^{2} V(r) n(r) \propto r^{1 / 2-\alpha}$, 

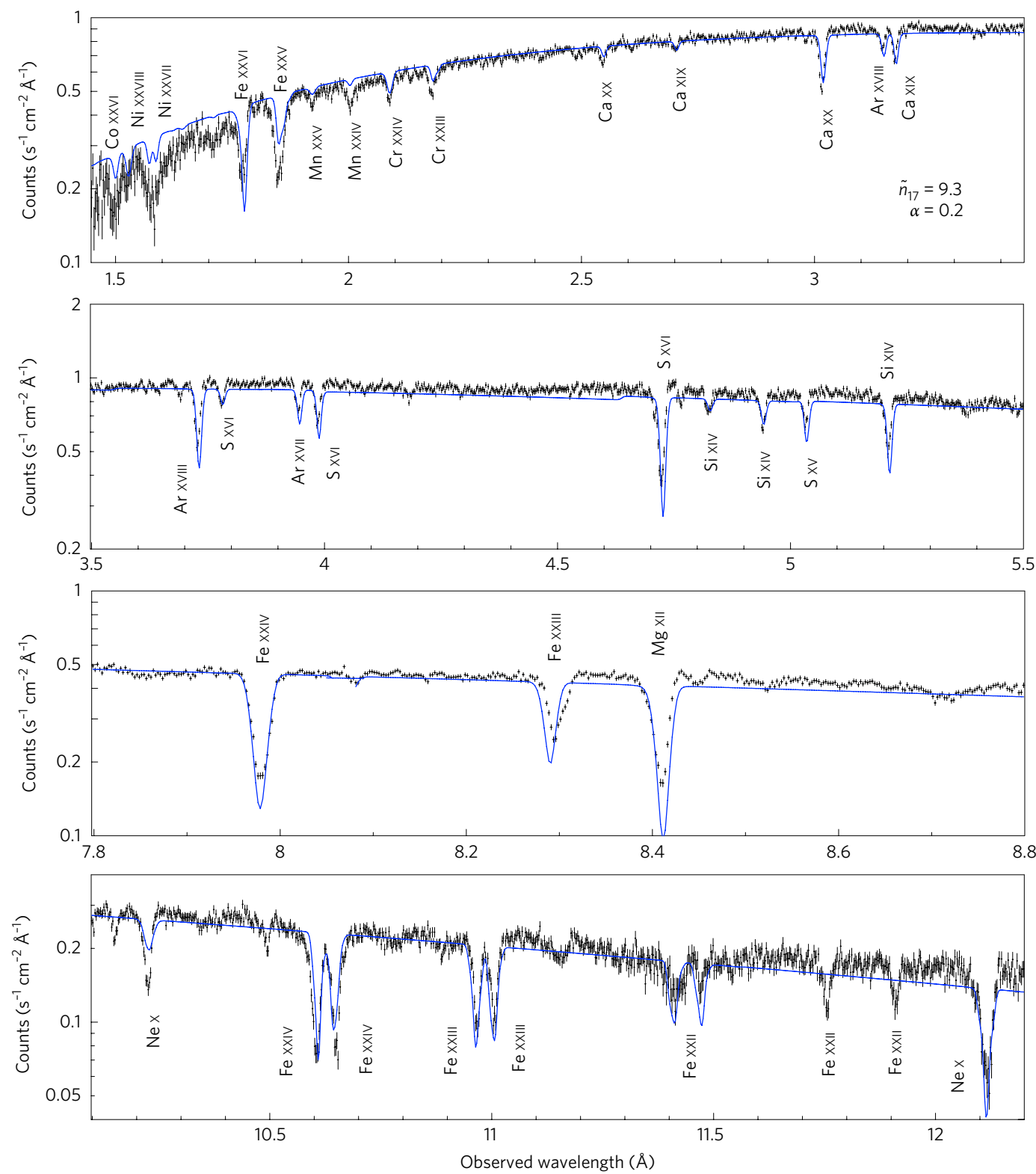

Figure 3 | The 46-ks Chandra/HETG spectrum of GRO J1655-40 overlaid on the global MHD wind model. Results are for $\alpha=0.2$ (blue line) with $\tilde{n}_{17}=9.3$. Adopting the previously constrained baseline continuum (a multicolour disk model diskbb of $k T=1.35 \mathrm{keV}$ with $L_{x}=5 \times 10^{37} \mathrm{erg} \mathrm{s}^{-1}$ ), the composite line spectra are computed assuming solar abundances for all ions as discussed in the text and shown in detail in Fig. 4. We find $\theta=80^{\circ}$ and $n_{0}=9.3 \times 10^{17} \mathrm{~cm}^{-3}$ at $r_{0}=3.81 \times 10^{6} \mathrm{~cm}$ with black hole mass $M=7 M_{\odot}$ for the best-fit spectrum. The best-fit wind parameters are listed in Supplementary Table 2.

is an increasing function of $r$ for $\alpha<1 / 2$. Therefore, most of the mass available for accretion escapes before reaching the black hole. There is mounting evidence that this is the case (wind mass flux similar to or higher than that needed to power the continuum) both in $\mathrm{AGNs}^{2}$ and in galactic X-ray binaries ${ }^{30}$.

\section{Methods}

Chandra/HETGS observation and data analysis. GRO J1655-40 was observed in its outburst phase at an exceptionally high cadence with Chandra/ HETGS on 2005 April 1, starting at 12:41:44 (UT or MJD 53461.53) yielding a total exposure of $44.6 \mathrm{ks}$ after the standard filtering. We started with the same spectral data extracted by the previous work ${ }^{7,17}$, in which a detailed data reduction procedure is described and used subsequently as described ${ }^{18}$ (and references therein). For subsequent analysis steps we used the heasoft v.6.19 package and the latest calibration files.

The standard gratings redistribution matrix file ( $\mathrm{rmfs}$ ) was used to generate its ancillary files (arfs). We used only the first-order dispersive data in this analysis. The net source count rate is $74.81 \mathrm{cts} \mathrm{s}^{-1}$, and the observed flux is $f_{2-10}=1.98 \times 10^{-8} \mathrm{erg} \mathrm{cm}^{-2} \mathrm{~s}^{-1}$ in $2-10 \mathrm{keV}$. All the spectra were backgroundsubtracted and dead-time-corrected following the standard procedures ${ }^{7,17}$.

Before applying our analysis, we examined the earlier X-ray studies ${ }^{7,17,18}$ to securely establish the continuum component in the observed broad-band spectrum. In agreement with the Rossi X-ray Timing Explorer (RXTE) hard $\mathrm{X}$-ray $(>2 \mathrm{keV})$ spectrum, we adopted the power law (po) model to describe a power-law continuum with photon index $\Gamma=3.54$ (fixed) with an appropriate normalization $K_{\mathrm{pl}}$. We then used a multicolour black body disk model (diskbb in XSPEC) with maximum disk temperature $k T=1.34 \mathrm{keV}$ (fixed) and normalization of $K_{\text {diskb }}$ to match the estimated disk luminosity. The latter component accounted for the dominant continuum flux. Therefore, throughout our analysis we have used spectral parameters identical with those used in the literature. The ionizing luminosity in GRO J1655-40 was taken to be $L_{\mathrm{X}}=5.0 \times 10^{37} \mathrm{erg} \mathrm{s}^{-1}$, in accordance with the references ${ }^{7,17,18}$, and the disk inclination angle $\theta$ was constrained to be in the range $67^{\circ}-85^{\circ 7,31}$. All the spectra were adaptively binned to ensure an appropriate minimum of counts per energy bin in order to perform the $\chi^{2}$ minimization in model fitting. Throughout, we include the Galactic absorption due to neutral gas of hydrogen-equivalent column $n^{7,17,18,32}$ of $N_{\mathrm{H}}=7.4 \times 10^{21} \mathrm{~cm}^{-2}$. 
a

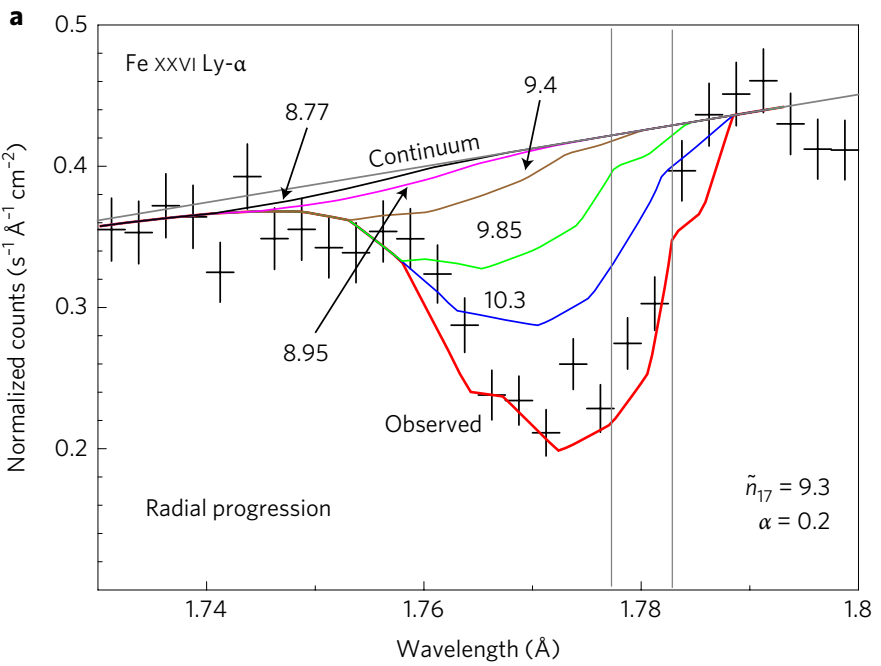

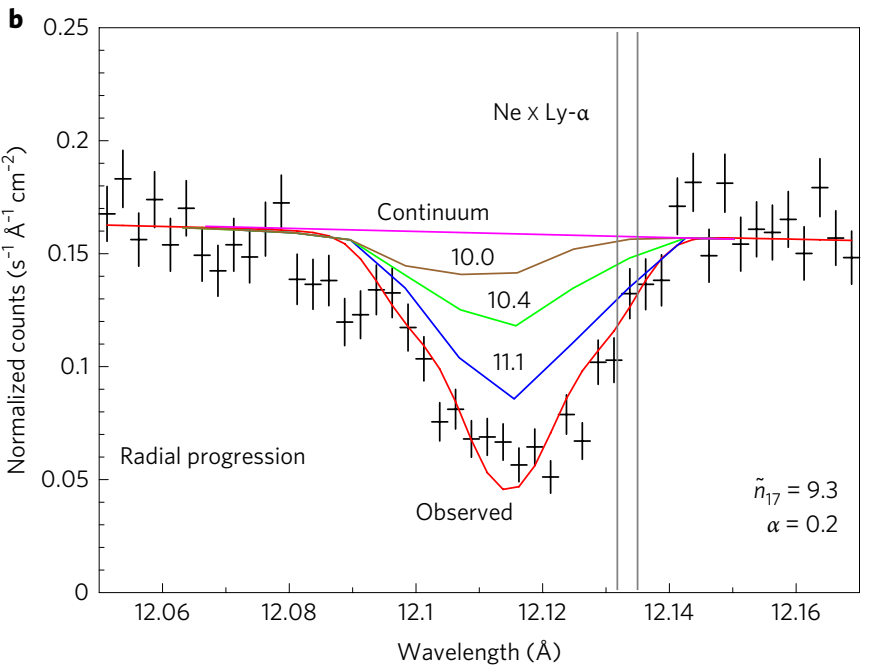

Figure 4 | Modelling the Fe xxvı Ly- $\alpha$ doublet $(1.778,17834 \AA$ ) and the $\mathrm{Ne} x$ Ly- $\alpha$ doublet $(12.132,12.136 \AA$ ) with the MHD winds of $\alpha=0.2$

and $\tilde{\mathbf{n}}_{\mathbf{1 7}}=\mathbf{9 . 3}$. The progressive development of each spectrum with increasing distance $r$ is shown by the sequence of coloured lines convolved with the Chandra/HETG resolution and overlaid on the data (the red line corresponds to the line profile as seen at infinity). The profiles were computed by using the values of $\left(n_{0}, \alpha, \theta\right)$ of Fig. 3. The double vertical lines denote the rest-frame wavelength of these transitions, while the number on each coloured line denotes the value of $\log r(\mathrm{~cm})$ along the line of sight. Note that the model spectra (in colour) are not fits to the data but an a priori calculation overlaid on GRO J1655-40 data.

This continuum spectral shape was then fixed in the subsequent analysis of the $\mathrm{X}$-ray absorbers to maintain consistency.

The MHD accretion-disk wind model. Motivated by the exceptional signal to noise ratio of the Chandra/HETGS data, we explored their interpretation within the framework of global MHD accretion-disk winds, which is primarily based on the well-defined MHD framework ${ }^{13}$, having been applied successfully to describe the main characteristics of AGN warm absorbers ${ }^{33-36}$ as well as the ultra-fast outflows of AGNs as diverse as Seyfert galaxies ${ }^{3}$ and bright quasars ${ }^{37}$. It is implemented here to model the ionized outflows associated with XRBs such as GRO J1655-40.

To this end, we assumed different scalings of the magnetic flux with radius while numerically solving the Grad-Shafranov equation within the ideal MHD approximation ${ }^{5,6,19,20}$. The radial profile of the wind density considered in this study is given by $n(r) \propto r^{-(1+\alpha)}$; we have explored four different cases, corresponding to $\alpha=-0.1,0,0.2$ and 0.4 , each representing a slightly different distinct global wind profile (see later description for details). We incorporated the wind structure into our radiative transfer formulation with radiation spectrum given by the SED of GRO J1655-40 (dominated by the thermal multicolour disk component, diskbb, of its high/soft state during the observation) and calculated its ionization equilibrium under heating-cooling balance in the wind using the XSTAR photoionization code (v.2.2.1bn21) $)^{29}$ in an approach similar to our previous works ${ }^{5,6,20,21,38,39}$. In the current wind model, we considered different sets of parameters as listed in Supplementary Table 1. The basic global MHD wind structure is shown in Supplementary Fig. 1: the poloidal magnetic field lines (thick solid) are plotted along with the 2D density distribution $n(r, \theta)$ (in colour), its contours (thin solid) and contours of the ionization parameter $\xi$ (dashed), as a function of cylindrical coordinates $(R, Z)$ in units of the innermost launching radius $r_{0}$. The density $n(r, \theta)$ is normalized to its maximum value. Note the linear $(Z)-\log (R)$ character of this figure, where the line of sight is not a straight line. Plasma in the wind is efficiently accelerated along each field line from the disk surface which is treated as a boundary condition in this study. The main acceleration phase takes place by the action of magnetic processes before the plasma becomes Alfvénic. The primary ionizing X-ray photons are produced in the innermost disk region which is treated as a point-like source in our radiative transfer calculations.

Implementing a total of approximately 80 line transitions as well as edges from a series of commonly observed elements ( $\mathrm{Ne}, \mathrm{Mg}, \mathrm{Si}, \mathrm{S}, \mathrm{Ar}, \mathrm{Ca}, \mathrm{Cr}, \mathrm{Mn}$, $\mathrm{Fe}, \mathrm{Co}, \mathrm{Ni}$ ) of various ionization states, our model provides a unique realization of the observed warm absorbers in GRO J1655-40 in a coherent context, in which each X-ray absorbing ion is explicitly associated with a specific segment of the same contiguous disk wind. In this approach, the emergence and properties of the different X-ray absorbing ions, from the soft band $(\lambda \approx 12.1 \AA)$ to the Fe $\mathrm{K}$ band $(\lambda \approx 1.5 \AA)$, are not independent of each other, but coupled and constrained by their common underlying wind structure (as they ought to be), reducing the arbitrariness in our calculations. Therefore, the proposed X-ray absorber model of GRO J1655-40 is not only internally consistent but also strongly constrained globally.

Our model is scale-invariant; thus the entire 2D wind structure is independent of the black hole mass for a given set of characteristic wind variables. These are $\mathrm{a}^{5,13}$ the particle-to-magnetic flux ratio $F_{0}$, the angular momentum $H_{0}$ and the initial launching angle $\theta_{0}$. These variables uniquely determine, accordingly, the rest of the dependent parameters ${ }^{5,13,19}$, such as the rotation of the magnetic field lines $\Omega_{0}$ and the specific Bernoulli function $J_{0}$. We choose $F_{0}=0.065, H_{0}=-1.7, \Omega_{0}=1.016$ and $J_{0}=-1.516$ in our fiducial model ${ }^{5,13}$. Our computational method applies the same approach used in our previous and other works $s^{5,38,39}$ : that is, we assume a geometrically thin accretion disk at the equator $(\theta=\pi / 2)$ as a boundary condition where accreting plasma is in Keplerian motion $\left(V_{\phi} \approx V_{\mathrm{K}} \propto r^{-1 / 2}\right)$. Owing to the poloidal field component and compression of the toroidal field, plasma is magneto-centrifugally launched by the $\mathbf{J} \times \mathbf{B}$ force from the disk surface with $\mathbf{V}=\left(V_{r}, V_{\theta}, V_{\phi}\right)=\left(0,0.01, V_{\mathrm{K}}\right)$, and it is efficiently accelerated along a streamline up to a few times the initial Keplerian velocity at large distances ${ }^{5,13}$. With the initial conditions on the disk (denoted by the subscript 0$)$ at $\left(r=r_{0} \approx r_{\text {ISCO }}\right.$, the innermost stable circular orbit, and $\left.\theta=\theta_{0}=\pi / 2\right)$, the density normalization at $\left(r_{0}, \theta_{0}\right)$ around a black hole (BH) of mass $M$ is expressed as

$$
n_{0} \equiv \frac{f_{\mathrm{w}} \dot{m}_{\mathrm{a}}}{\sigma_{\mathrm{T}} R_{\mathrm{S}}}=\tilde{n}_{17}\left(f_{\mathrm{w}}, \dot{m}\right)\left(\frac{M}{10 M_{\odot}}\right)^{-1} \mathrm{~cm}^{-3}
$$

where $\sigma_{\mathrm{T}}$ is the Thomson cross-section, $\dot{m}_{\mathrm{a}}$ is the dimensionless mass-accretion rate, $\tilde{n}_{17}$ is the wind density factor in units of $10^{17} \mathrm{~cm}^{-3}$, and $f_{\mathrm{w}}$ is the ratio of the outflow rate in the wind to accretion at $r=r_{0} \approx r_{\text {ISCO }}$ for the black hole mass $M=7 M_{\odot}$ relevant for GRO J1655-40. The exact values for these quantities, however, depend on the specific source and its specific spectral state. In our model, we let $\tilde{n}_{17}$ vary also, among other parameters, to search for the best-fit spectrum as found in Figs 3 and 4. Then, a global density distribution on the $(r, \theta)$-plane is determined by

$$
n(r, \theta)=n_{0} g(r) f(\theta)
$$

where the angular dependence $f(\theta)$ is numerically solved by the Grad-Shafranov equation (the momentum-balance equation in a direction perpendicular to a field line) in an ideal MHD framework. For the observed ${ }^{9-11} L_{\text {ion }}=5.0 \times 10^{37} \mathrm{erg} \mathrm{s}^{-1}$, one can estimate that

$$
\xi(r, \theta) \equiv \frac{L_{\text {ion }}}{n(r, \theta) r^{2}} \operatorname{erg~} \mathrm{cm} \mathrm{s}^{-1}
$$

for a given set of $\left(\tilde{n}_{17}, \theta, \alpha\right)$ as listed in Supplementary Table 1.

$\mathrm{X}$-ray photoionization of disk winds. The definition of the photoionization parameter $\xi$, which determines the wind plasma ionization, involves the local 
radiation field, which is in general different from that of the input SED because of absorption along the wind. The correct study of wind ionization therefore requires that the input SED be transferred correctly to each wind point numerically. To this end, we performed radiative transfer calculations of the input X-ray spectrum, using $\mathrm{XSTAR}^{29}$ to compute the the local plasma ionization and resulting opacity as a function of the wavelength along a given line of sight. Using the ionizing spectrum of the baseline continuum of GRO J1655-40 described above, we discretize the wind along a given line of sight of angle $\theta$ into a large number of contiguous zones of logarithmically constant width $\Delta r / r \approx 0.15$, sufficiently small to consider each radial zone as plane-parallel ${ }^{5}$. Having obtained the ionic column $N_{\text {ion }}$ appropriate for each line transition from the photoionization calculations, one can compute line spectra through the local line optical depth

$$
\tau_{\nu}(r, \theta) \equiv \sigma_{\text {photo, } \nu} N_{\text {ion }}
$$

where $\sigma_{\text {photo, } \nu}$ is the line photoabsorption cross-section at frequency $\nu$ calculated by assuming a Voigt profile for the transition with the local wind shear $\mathrm{d} V / \mathrm{d} r$ for line broadening in place of the plasma turbulent velocity ${ }^{40}$ and integrating the local absorption over all distances along the line of sight.

In the commonly used phenomenological fitting analyses, the absorbers across the wavelength range are typically treated as mutually independent components arising from physically disconnected regions. In contrast, the proposed model must and does account for all the absorbers simultaneously from the same continuous global disk wind. In the end, we implement our MHD-wind model, mhdabs, into XSPEC as a multiplicative table model. The symbolic spectral form reads as

$$
\text { tbabs } \times(\text { po }+ \text { diskbb }) \times \text { mtable }\{\text { mhdabs }\}
$$

where we have used the previously estimated values of parameters such as the Galactic absorption due to neutral hydrogen column (tbabs) ${ }^{32}, N_{\mathrm{H}}=7.4 \times 10^{21} \mathrm{~cm}^{-2}$, and the black hole mass of $M=7 M_{\odot}$.

Physical parameters of magnetically driven X-ray absorbers. The present study takes a straightforward, yet physically motivated, approach to modelling the properties of X-ray absorbers of GRO J1655-40 within the framework of a well defined class (MHD) of accretion disk wind models. Figure 3 shows the $46-\mathrm{ks}$ Chandra/HETG broadband spectrum of GRO J1655-40 between $~ 1.5$ and $12 \AA$ with the best-fit mhdabs model of $\alpha=0.2$ and $\tilde{n}_{17}=9.3$; in all our calculations we assume solar ion abundances $\left(A_{\text {ion }}=1\right)$ for all elements, as the simplest, least contrived assumption. In producing Fig. 3 as well as all similar figures, we let the wind density normalization $n_{0}$ (thus $\tilde{n}_{17}$ ) vary along with the value for $\alpha$ in a way that the model produces the correct value of most ionic columns. The disk inclination angle $\theta$ was also allowed to vary within the narrow angle range allowed by observation and was set to $80^{\circ}$ in all figures. We present the dependence of individual lines on the wind density normalization $\tilde{n}_{17}$ for $\alpha=0.2$ in Supplementary Fig. 2 . The global spectral fit at longer (Fe xxiI near $\sim 11 \AA$ ) and shorter (Ca XIx. Ca Xx and Ar xviII near $3.1 \AA$ ) wavelengths clearly favours $\tilde{n}_{17}=9.3$. Detailed absorbers' properties are listed in Supplementary Table 2 . We see that the model captures correctly most of the features of the spectrum. Discrepancies in a few transitions could be attributed either in different abundances or in the fact that the wind density might deviate locally from the smooth, contiguous power-law dependence assumed throughout this work.

Besides the spectra for different values of $\alpha$, we also present the ratios of the observed to modelled line equivalent widths (EW) for major ions, as done in other related work ${ }^{7,24}$, along with their mean (solid green) and median (dotted green) values also indicated by numbers (see Supplementary Figs 3 and 4). We have further calculated the mean values of their relative deviations $\Delta_{\mathrm{EW}}=\mid(\mathrm{EW}(\mathrm{obs})-\mathrm{EW}($ model $)) /$ $\mathrm{EW}$ (model) and we found the following pairs of values $\left(\alpha, \Delta_{\mathrm{EW}}\right)=(-0.1,0.54)$, $(0.0,0.44),(0.2,0.24),(0.4,0.47)$. The ratios here serve as a measure of the goodness of the global fit for our calculations. With this measure, we have demonstrated that the $\alpha=0.2$ wind (with the best-fit value of $\tilde{n}_{17}=9.3$ ) shown in Fig. 3 is indeed statistically favoured over the other wind structures in support for our conclusion.

Mass and energy budget of X-ray winds in GRO J1655-40. The MHD winds that are launched from an accretion disk as discussed above, can carry power comparable to or larger than their radiant power and mass flux larger than that needed to power their luminosity by accretion. These can have significant influence on their surroundings, especially in the $\mathrm{AGNs}^{41}$ that are located at the centres of galaxies or clusters. These winds are also of interest in XRBs for determining the energy budget of the system.

One generally estimates the mass flux and luminosity associated with a specific transition of a known $\xi$-value and measured velocity $V$. Because

$$
\dot{M}_{\text {out }}^{(\text {local })} \approx n(r) r^{2} V \text { and } \xi=\frac{L_{\mathrm{X}}}{n(r) r^{2}} \text {, then } \dot{M}_{\text {out }}^{(\text {local })} \approx \frac{L_{\mathrm{X}}}{\xi} V
$$

which is a general relation, applicable to any transition of known $\xi$ and measured $V$. For winds with density scaling given by our model, $\xi$ is proportional to $r^{-(1+\alpha)}$ and $V \propto r^{-1 / 2}$, leading to

$$
\dot{M}_{\text {out }}^{(\text {local })} \approx n(r) r^{2} V \propto r^{1 / 2-\alpha}
$$

indicating that for $\alpha<1 / 2$, the mass flux in the wind increases with distance ${ }^{8,13}$. So, with the above scalings, although there is mass flux of fully ionized plasma (not discernible in the spectrum) at radii smaller than those at which the Fe features occur (namely at $r \gtrsim 10^{4} R_{\mathrm{S}}$ ), this mass flux is a small fraction of that associated with the partially ionized plasma. For the value of $\tilde{n}_{17}$ appropriate to our best fit of the data, we obtain $\dot{M} \sim 10^{17}\left(r / R_{S}\right)^{1 / 2-\alpha} \mathrm{g} \mathrm{s}^{-1}$, the value of which, for $r \approx R_{\mathrm{S}}$, is comparable to that needed to produce the X-ray luminosity of this source. This is consistent with a value $f_{\mathrm{w}} \approx 1$ for the parameter of equation (1) that determines the ratio of the accreted to wind mass flux near the disk inner edge, in that it reproduces the observed radiation with efficiency of $\sim 0.1$.

Finally, one can further estimate the corresponding kinetic power due to mass loss, because the kinetic power scales as

$$
\dot{E}_{\text {out }}^{(\text {local })} \propto \dot{M}_{\text {out }}^{(\text {local })} v_{\text {out }}^{2} \propto r^{-(\alpha+1 / 2)} .
$$

This expression indicates that, for the scalings of our model, even though most mass loss comes from the largest wind radii, most of the wind kinetic energy comes from the wind segments launched in the proximity of the black hole. With the best-fit value of $n_{0}$ derived from $\approx$ our analysis, the wind kinetic luminosity is of the order of $\dot{E}_{\text {kin }} \lesssim 10^{38} \mathrm{erg} \mathrm{s}^{-1}$, comparable with its photon luminosity and broadly consistent with the fact that for $r \approx R_{\mathrm{S}}$ the mass flux in the wind is comparable to that of accretion. Considering that the Fe transitions are formed at radii $r \gtrsim 10^{4} R_{\mathrm{S}}$, the kinetic luminosity attributed to the Fe emitting plasma is smaller by a factor of $R_{\mathrm{S}} / r \approx 10^{-4}$ or $\dot{E}_{\mathrm{kin}, \mathrm{Fe}} \approx 10^{33} \mathrm{erg} \mathrm{s}^{-1}$. One should note that for a given $\dot{m}$ all winds carry the same kinetic power per unit black hole mass, $\dot{E}_{\text {out }} / M$. However, because of their higher ionization and formation of the Fe xxv / Fe xxvi absorbers at larger (normalized) distances, the $\dot{E}_{\text {out }} / M$ of XRBs will be smaller than those of AGN, as documented in a recent work ${ }^{42}$.

Assessment of the different wind density profiles. Supplementary Fig. 3 gives the broadband spectra of our models for the different values of $\alpha$ for which $\tilde{n}_{17}$ is constrained by the broadband fitting. As discussed earlier, successful models should provide the correct $\left(N_{\mathrm{H}}, V\right)$ at the values of $\xi$ that produce a given ionic species $^{43}$. Clearly, such a fit is by necessity statistical given the broad range of $\xi$ at which the various ions of the spectrum appear. Our density parametrization allows a global view of this notion. The optimal value of $\alpha$ was chosen so that it minimizes the differences between observed and computed equivalent width of the ensemble of the transitions by varying $\tilde{n}_{17}$ (see Supplementary Fig. 4 ) in the fitting procedure. Changing $\tilde{n}_{17}$ for a given $\alpha$ thus minimizes deviations of calculation from observation at the band associated with given value of $\xi$, to which the particular choice is tailored (see also Supplementary Fig. 2 for the significance of $\tilde{n}_{17}$ ). However, the wrong value of $\alpha$ then produces increasingly large deviations from observations either at lower or larger $\xi$. So we see that for $\alpha=-0.1$ (green) we underestimate the equivalent width of short-wavelength transitions, whereas for $\alpha=0.4$ (orange) the low-ionization transitions have very small columns, excluding this value. Thus, Supplementary Figs 3 and 4 combined together clearly indicate that the model with $\alpha=0.2$ is preferred by these metrics at a statistically significant level.

Clearly, better fits to the data can be achieved by judiciously introducing breaks in the wind density profile to provide better fits to the local plasma column. Such breaks are not excluded by any underlying principle but only by the self-similarity requirement of our solutions. However, we have chosen not to do so. Such an approach might be the subject of future investigations.

In closing, we would like to draw attention to another recent work on XRB MHD winds ${ }^{43}$ that studied the photoionization of the accretion disk winds of the similar MHD-driven model ${ }^{15}$ as they relate to the absorbers of GRO J1655-40. These winds have steeper density profiles $(\alpha \gtrsim 0.4)$ than those used herein and in our earlier AGN work; as such, the values of $\xi$ that allow for the presence of Fe XXvi (used as a measure of the wind ionization) occur at larger distances than in the winds used herein. Given the steeper density profiles, the corresponding columns are smaller than observation implies (see our Supplementary Figs 3 and 4), while in the absence of detailed absorption line modelling it is uncertain how well that model would fit the data. On the other hand, their wind models relate the density and magnetic flux distribution more closely to the dynamics of the accretion disk than those of our model, through a balance between field accretion and diffusion. We plan to address this issue, as it relates to the winds we have so far used, in a future work.

Data availability. The data that support the plots within this paper and other findings of this study are available from the corresponding author upon reasonable request.

\section{Received 26 July 2016; accepted 24 January 2017; published 6 March 2017}

\section{References}

1. Crenshaw, D. M., Kraemer, S. B. \& George, I. M. Mass loss from the nuclei of active galaxies Annu. Rev. Astron. Astrophys. 41, 117-167 (2003). 
2. Behar, E. et al. A long look at NGC 3783 with the XMM-Newton reflection grating spectrometer. Astrophys. J. 598, 232-241 (2003).

3. Tombesi, F. et al. Evidence for ultra-fast outflows in radio-quiet AGNs. I. Detection and statistical incidence of Fe K-shell absorption lines. Astron. Astrophys. 521, 57-92 (2010).

4. Holczer, T., Behar, E. \& Kaspi, S. Absorption measure distribution of the outflow in IRAS 13349+2438: direct observation of thermal instability? Astrophys. J. 663, 799-807 (2007).

5. Fukumura, K., Kazanas, D., Contopoulos, I. \& Behar, E. Magnetohydrodynamic accretion disk winds as X-ray absorbers in active galactic nuclei. Astrophys. J. 715, 636-650 (2010).

6. Fukumura, K., Kazanas, D., Contopoulos, I. \& Behar, E. Modeling highvelocity QSO absorbers with photoionized magnetohydrodynamic disk winds. Astrophys. J. Lett. 723, L228-L232 (2010).

7. Miller, J. M. et al. The magnetic nature of disk accretion onto black holes. Nature 441, 953-955 (2006).

8. Blandford, R. D. \& Begelman, M. C. On the fate of gas accreting at a low rate on to a black hole. Mon. Not. R. Astron. Soc. 303, L1-L5 (1999).

9. Tombesi, F. et al. Wind from the black-hole accretion disk driving a molecular outflow in an active galaxy. Nature 519, 436-438 (2015).

10. Begelman, M. C., McKee, C. F. \& Shields, G. A. Compton heated winds and coronae above accretion disks. I. Dynamics. Astrophys. J. 271, $70-88$ (1983).

11. Murray, N. et al. Accretion disk winds from active galactic nuclei. Astrophys. J. 451, 498 (1995).

12. Blandford, R. D. \& Payne, D. G. Hydromagnetic flows from accretion discs and the production of radio jets. Mon. Not. R. Astron. Soc 199, 883-903 (1982).

13. Contopoulos, J. \& Lovelace, R. V. E. Magnetically driven jets and winds: exact solutions. Astrophys. J. 429, 139-152 (1994).

14. Contopoulos, J. A simple type of magnetically driven jets: an astrophysical plasma gun. Astrophys. J. 450, 616-627 (1995).

15. Ferreira, J. Magnetically-driven jets from Keplerian accretion discs. Astron. Astrophys. 319, 340-359 (1997).

16. Neilsen, J. \& Homan, J. A hybrid magnetically/thermally driven wind in the black hole GRO J1655-40? Astrophys. J. 750, 27-35 (2012).

17. Miller, J. M. et al. The accretion disk wind in the black hole GRO J1655-40. Astrophys. J. 680, 1359 (2008).

18. Kallman, T. R. et al. Spectrum synthesis modeling of the X-ray spectrum of GRO J1655-40 taken during the 2000 outburst. Astrophys. J. 701, 865-884 (2009).

19. Luketic, S., Proga, D., Kallman, T. R., Raymond, J. C. \& Miller, J. M. On the properties of thermal disk winds in X-ray transient sources: a case study of GRO J1655-40. Astrophys. J. 719, 515-522 (2010).

20. Fukumura, K. et al. Magnetically driven accretion disk winds and ultra-fast outflows in PG 1211+143. Astrophys. J. 805, 17-27 (2015)

21. Kazanas, D., Fukumura, K., Behar, E., Contopoulos, I. \& Shrader, C. Toward a unified AGN structure. Astron. Rev. 7, 92-123 (2012).

22. Shidatsu, M., Done, C. \& Ueda, Y. An optically thick disk wind in GRO J1655-40? Astrophys. J. 823, 159-171 (2016).

23. Neilsen, J., Rahoui, F., Homan, J. \& Buxton, M. A super-Eddington, Compton-thick wind in GRO J1655-40? Astrophys. J. 822, 20-34 (2016).

24. Netzer, H. A thermal wind model for the X-ray outflow in GRO J1655-40. Astrophys. J. Lett. 652, L117-L120 (2006).

25. Castor, J. I., Abbot, D. C. \& Klein, R. I. Radiation-driven winds in Of stars. Astrophys. J. 195, 157-174 (1975).

26. Proga, D., Stone, J. M. \& Kallman, T. R. Dynamics of line-driven disk winds in active galactic nuclei. Astrophys. J. 543, 686-696 (2000).

27. Behar, E. Density profiles in Seyfert outflows. Astrophys.J. 703, 1346-1351 (2009).

28. Miller, J. M. et al. Powerful, rotating disk winds from stellar-mass black holes. Astrophys.J. 814, 87-114 (2015).

29. Kallman, T. R \& Bautista, M. A. Photoionzation and high density gas. Astrophys. J. Suppl. 133, 221-253 (2001).

30. Neilsen, J. \& Lee, J. C. Accretion disk winds as the jet suppression mechanism in the microquasar GRS 1915+105. Nature 458, 481-484 (2009).
31. Hjellming, R. M. \& Rupen, M. P. Episodic ejection of relativistic jets by the X-ray transient GRO J1655-40. Nature. 375, 464-468 (1995).

32. Dickey, J. M. \& Lockman, F. J. H I in the Galaxy. Annu. Rev. Astron. Astr. 28, 215-261 (1990).

33. Blustin, A. J., Page, M. J., Fuerst, S. V., Branduardi-Raymont, G. \& Ashton, C. E. The nature and origin of Seyfert warm absorbers. Astron. Astrophys. 431, 111-125 (2005).

34. George, I. M. et al. ASCA observations of Seyfert 1 galaxies. III. The evidence for absorption and emission due to photoionized gas. Astrophys. J. Suppl. 114, 73-120 (1998).

35. McKernan, B., Yaqoob, T. \& Reynolds, C. S. A soft X-ray study of type I active galactic nuclei observed with Chandra high-energy transmission grating spectrometer. Mon. Not. R. Astron. Soc. 379, 1359-1372 (2007).

36. Laha, S. et al. Warm absorbers in X-rays (WAX), a comprehensive highresolution grating spectral study of a sample of Seyfert galaxies. I. A global view and frequency of occurrence of warm absorbers. Mon. Not. R. Astron. Soc., 441, 2613-2643 (2014).

37. Chartas, G., Saez, C., Brandt, W. N., Giustini, M. \& Garmire, G. P. Confirmation of and variable energy injection by a near-relativistic outflow in APM 08279+5255. Astrophys. J. 706, 644-656 (2009).

38. Schurch, N. J. \& Done, C. The impact of accretion disc winds on the X-ray spectrum of AGN. I. XSCORT. Mon. Not. R. Astron. Soc. 381, 1413-1425 (2007)

39. Sim, S. A., Long, K. S., Miller, L. \& Turner, T. J. Multidimensional modelling of X-ray spectra for AGN accretion disc outflows. Mon. Not. R. Astron. Soc. 388, 611-624 (2008).

40. Mihalas, D. Stellar Atmospheres (Freeman, 1978).

41. Crenshaw, D. M. \& Kraemer, S. B. Feedback from mass outflows in nearby active galactic nuclei. I. Ultraviolet and X-ray absorbers. Astrophys. J. 753, 75-85 (2012)

42. King, A. L. et al. Regulation of black hole winds and jets across the mass scale. Astrophys. J. 762, 103-121 (2013).

43. Chakravorty, S. et al. Absorption lines from magnetically driven winds in X-ray binaries. Astron. Astrophys. 589, A119-A135 (2016).

\section{Acknowledgements}

We thank T. Kallman for providing us with the Chandra/HETG data for GRO J1655-40. K.F., D.K. and C.S. acknowledge support by a NASA/ADP grant. E.B. received funding from the European Unions Horizon 2020 research and innovation programm under the Marie Sklodowska-Curie grant agreement no. 655324. and from the I-CORE program of the Planning and Budgeting Committee (grant number 1937/12). Support for this work was in part provided by NASA through Chandra Award Number AR6-17013A issued by the Chandra X-ray Observatory Center, which is operated by the Smithsonian Astrophysical Observatory for and on behalf of NASA under contract NAS8-03060.

\section{Author contributions}

K.F. led the overall model development and data fitting procedures. D.K., C.S, E.B. and F.T. each contributed to the validation, interpretation and presentation of the final results. The baseline wind model was originally formulated by I.C. In particular, D.K provided insights connecting our results to the broader picture of accretion-induced outflows in astrophysical environments and E.B. provided technical advice on the details of atomic physics crucial to the $\mathrm{X}$-ray spectroscopic analyses in this work. All authors contributed to writing the manuscript and preparing the figures and tables.

\section{Additional information}

Supplementary information is available for this paper.

Reprints and permissions information is available at www.nature.com/reprints.

Correspondence and requests for materials should be addressed to K.F.

How to cite this article: Fukumura, K. et al. Magnetic origin of black hole winds across the mass scale. Nat. Astron. 1, 0062 (2017).

\section{Competing interests}

The authors declare no competing financial interests. 